\title{
Bortezomib-inducible long non-coding RNA myocardial infarction associated transcript is an oncogene in multiple myeloma that suppresses miR-29b
}

\author{
Yunfeng Fu', Xiao Liu', Fangrong Zhang ${ }^{1}$, Siyi Jiang ${ }^{1}$, Jing Liu' ${ }^{1}$ and Yanwei Luo ${ }^{1}$
}

\begin{abstract}
Clinical outcomes of patients with multiple myeloma (MM) have almost doubled the overall survival over the last decade owing to the use of proteasome inhibitor such as bortezomib (BTZ). However, some patients with MM develop primary resistance to $B T Z$, whereas others develop resistance after treatment. In this study, we investigated relationships between BTZ resistance and dysfunction of long non-coding RNAs (IncRNAs) in patients with MM. Bone marrow samples were collected from patients with MM and healthy donors for IncRNA microarray and survival analyses. To investigate functions and underlying mechanisms of IncRNA-mediated BTZ resistance in MM, we performed CCK-8 assays, flow cytometry analyses, dual luciferase report gene assays, and RNA pulldown assays with samples from nude mice carrying tumor xenografts and in clinical samples. Differentially expressed IncRNA myocardial infarction associated transcripts (MIAT) were highly expressed in patients with MM compared with healthy controls, and were predictive of poor survival outcomes. Moreover, MIAT expression was significantly increased in BTZ-resistant patients with MM compared with newly diagnosed patients with MM, and was identified as a BTZ-inducible IncRNA. Specifically, BTZ upregulated MIAT expression through increased stat1 phosphorylation. Silencing of MIAT inhibited MM cell growth and sensitized MM cells to BTZ by negatively regulating miR-29b. Our data demonstrated the utility of MIAT as a tool for overcoming BTZ resistance in patients with MM.
\end{abstract}

\section{Introduction}

Multiple myeloma (MM) accounts for approximately $10 \%$ of hematological malignancies ${ }^{1}$. Over the past decade, treatments with second-generation proteasome inhibitors, such as bortezomib (BTZ) and carfilzomib, have improved clinical outcomes for patients with MM and almost doubled overall survival ${ }^{2}$. Myeloma cells are

Correspondence: Jing Liu (jjliuliuyswq@126.com) or Yanwei Luo (516534169@qq.com)

${ }^{1}$ The Third Xiangya Hospital of Central South University, Changsha 410013, China

These authors contributed equally: Yunfeng Fu, Xiao Liu

Edited by F. Sesti sensitized to the inhibition of the $26 \mathrm{~S}$ proteasome, resulting in the inhibition of NF- $\mathrm{kB}$ signaling ${ }^{3}$. However, owing to primary and acquired resistance to BTZ, most patients suffer relapse following treatment ${ }^{4}$. To address this challenge, genome-wide transcription studies of MM have identified multiple biomarkers that can be used for personalized treatments, including non-coding RNAs.

Long non-coding RNAs (lncRNAs) comprising more than 200 nucleotides ${ }^{5}$ participate in multiple biological processes involving epigenetic alterations. Moreover, dysfunctions of lncRNAs are associated with tumorigenesis and drug resistance in various cancers, including $\mathrm{MM}^{6}$. In particular, the IncRNA MALAT1 is overexpressed in $\mathrm{MM}$ and may be predictive of tumor

\section{(c) The Author(s) 2019}

(c) (i) Open Access This article is licensed under a Creative Commons Attribution 4.0 International License, which permits use, sharing, adaptation, distribution and reproduction c. in any medium or format, as long as you give appropriate credit to the original author(s) and the source, provide a link to the Creative Commons license, and indicate if changes were made. The images or other third party material in this article are included in the article's Creative Commons license, unless indicated otherwise in a credit line to the material. If material is not included in the article's Creative Commons license and your intended use is not permitted by statutory regulation or exceeds the permitted use, you will need to obtain permission directly from the copyright holder. To view a copy of this license, visit http://creativecommons.org/licenses/by/4.0/. 
progression $^{7,8}$. Similarly, lncRNAs, such as CCAT1, H19, and NEAT1, have potential as biomarkers and treatment targets in patients with $\mathrm{MM}^{9-11}$, and NEAT1 knockdown reportedly improved dexamethasone sensitivity in patients with $\mathrm{MM}^{10}$. Moreover, Lu et al. recently showed that Linc00515 confers chemoresistance to melphalanresistant myeloma cells by inhibiting miR-140-5 $\mathrm{p}^{12}$. These studies warrant assessments of the roles of IncRNAs in BTZ resistance of MM.

In the current study, we identified differentially expressed lncRNAs in patients with MM. Among these, the lncRNA myocardial infarction associated transcript (MIAT) was highly expressed in patients with MM compared with healthy controls. Thus, we determined expression levels of MIAT in MM cells and the association of MIAT and prognosis of patients with MM were also investigated. In subsequent experiments, shRNAmediated knockdown of MIAT sensitized MM cells to BTZ by regulating miR-29b. Our findings suggest that MIAT inhibition has potential as a therapeutic strategy for overcoming acquired BTZ resistance in patients with MM.

\section{Material and methods}

\section{Tissue samples}

Three MM and three healthy donor control bone marrow samples were collected for microarray analyses. Bone marrow tissues were obtained from an independent cohort of 143 patients with MM with clinical staging and survival information, 46 with newly diagnosed MM (NDMM), 34 with relapsed/refractory MM (RRMM), 35 with smoldering $\mathrm{MM}$ (SMM), and 28 with extramedullary myeloma (EMM) and 56 healthy donors. All tissue samples and corresponding clinical data were used in qPCR and survival analyses. Informed consent was obtained from each patient. This project was approved by the Ethics Committee of The Third Xiangya Hospital of Central South University.

\section{IncRNA microarray analysis}

CD138+ plasma cells were collected from three patients with MM and three healthy donors (clinicopathological variables are shown in Table 1) and total RNA was extracted using RNeasy Mini Kits (Qiagen, GmBH, Hilden, Germany) according to the manufacturer's

Table 1 Clinicopathological variables of patients with multiple myeloma and healthy donor used in microarray assay

\begin{tabular}{|c|c|c|c|c|c|c|c|c|c|c|c|}
\hline Subjects & Age & Gender & Cytogenetic risk & Deletion 13q (\%) & Deletion 17p (\%) & t.(11.14) (\%) & t.(14·16). (\%) & DSS & ISS & $\lg \mathrm{H}$ & IgL \\
\hline MM patient 1 & 35 & Male & High & Yes & No & No & No & I & । & $\lg G$ & K \\
\hline MM patient 2 & 48 & Male & High & No & No & No & Yes & $\|$ & $\|$ & $\lg A$ & $\lambda$ \\
\hline MM patient 3 & 55 & Male & High & No & Yes & No & No & III & III & $\lg G$ & $\lambda$ \\
\hline Healthy donor 1 & 32 & Male & - & No & No & No & No & - & - & - & - \\
\hline Healthy donor 2 & 45 & Male & - & No & No & No & No & - & - & - & - \\
\hline Healthy donor 3 & 58 & Male & - & No & No & No & No & - & - & - & - \\
\hline
\end{tabular}

DSS Durie-Salmon staging, ISS international staging system

Table 2 The primer sequences used in qPCR

\begin{tabular}{|c|c|c|}
\hline Gene & Sense $\left(5^{\prime}-3^{\prime}\right)$ & Anti-sense $\left(5^{\prime}-3^{\prime}\right)$ \\
\hline MIAT & TCCCATTCCCGGAAGCTAGA & GAGGCATGAAATCACCCCCA \\
\hline miR-29b & GCGTAGCACCATTTGAAATC & CAGTGCGTGTCGTGGAGT \\
\hline miR-489 & CTCAACTGGTGTCGTGGAGTC GGCAATTCAGTTGAGAGCTGC CGT & ACACTCCAGCTGGGGTGACATCACATA \\
\hline miR-150 & GAGGATCCCCGGGTACCGGTCTGGCAGGAACCCCCGCCCT & CACACATTCCACAGGCTAGTAAAAGCCGCA GCAGAGATG \\
\hline Mcl-1 & GGACATCAAAAACGAAGACG & GCAGCTTTCTTGGTTTATGG \\
\hline CDK-6 & CCGAGTAGTGCATCGCGATCTAA & CTITGCCTAGTTCATCGATATC \\
\hline PSME4 & GGAGACCTTCTGCACTTCCAAGGATCTCAT & CCTCCCAAGTGTCTAAAGCCGCTTATACTG \\
\hline SP-1 & CCATACCCCTTAACCCCG & GAATTTCACTAATGTTTCCCACC \\
\hline U6 & CTCGCTTCGGCAGCACA & AACGCTTCACGAATTTGCGT \\
\hline$\beta$-actin & GCCCTATAAAACCCAGCGGC & TCGATGGGGTACTTCAGGGT \\
\hline
\end{tabular}




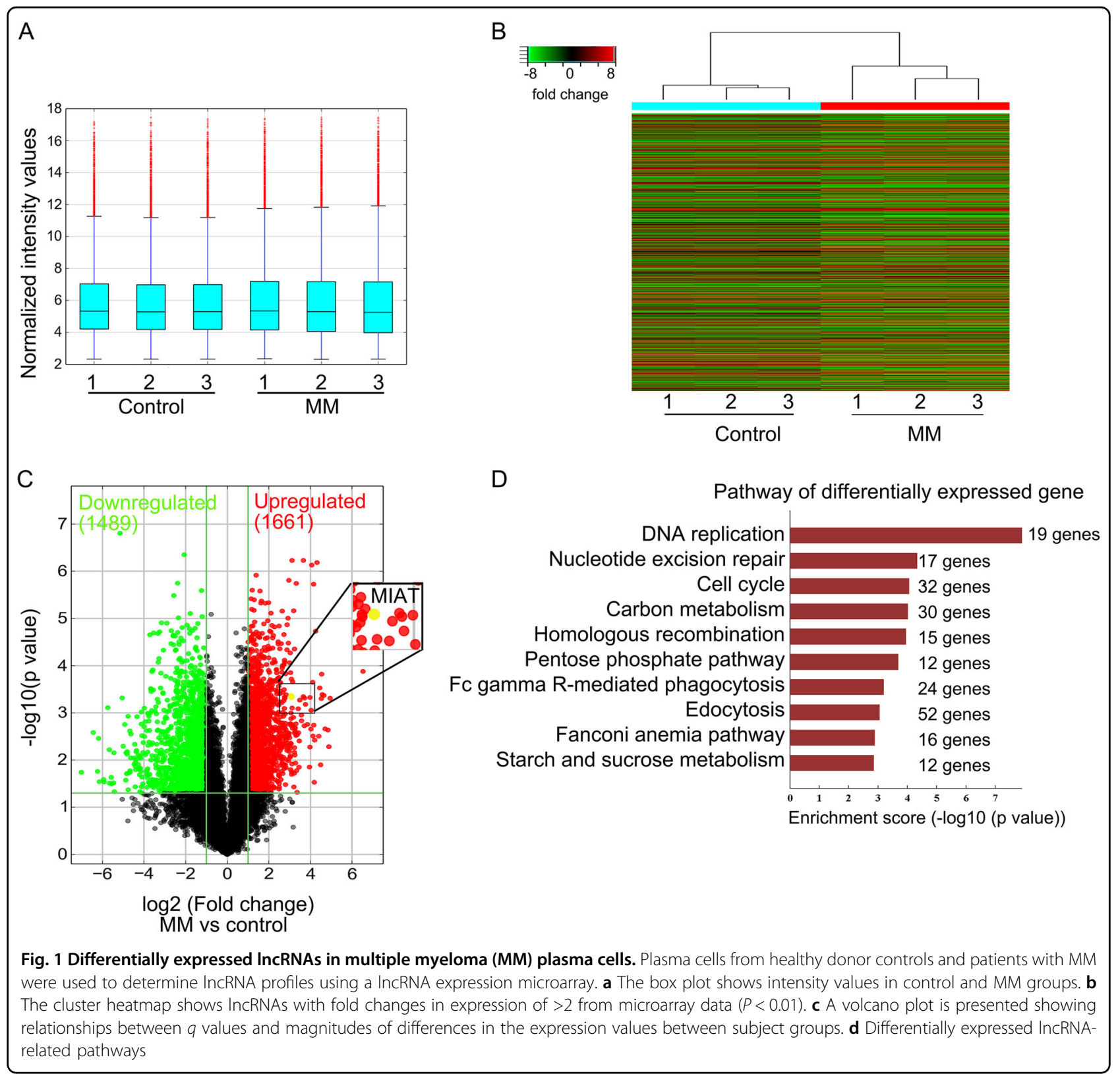

instructions. After purifying total RNA, IncRNA expression profiles were analyzed by Aksomics Co. Ltd. (Shanghai, China) using human lncRNA Array V4.0 ( $8 \times$ $60 \mathrm{~K}$ ), which includes 35,923 lncRNAs and 24,881 coding genes. Raw data were then analyzed using GenePix 4000B and Gene-Spring software. Differentially expressed lncRNAs were identified as those with fold changes of $\geq 2$ and $q$ values of $<0.05$.

\section{Cell cultures and treatments}

Myeloma cell lines (U266, KMS12, and KM3) were obtained from the National Infrastructure of Cell Line Resource (Beijing, China) and were cultured in RP1640 medium supplemented with $10 \%$ fetal bovine serum in an incubator containing $5 \% \mathrm{CO}_{2}$ at $37^{\circ} \mathrm{C}$. A BTZ-resistant U266 cell line (U266/BTZ) was established in our lab by treating $1 \times 10^{5}$ cells $/ \mathrm{ml}$ with 1 -nM BTZ. Media were changed once every 3 days and BTZ contents were maintained for 2 weeks, and were then doubled. After several iterations of dose doubling, cells were finally incubated in 30-nM BTZ.

Myeloma cell lines were exposed to BTZ at 0, 20, 40, and $80 \mathrm{nM}$ for $24 \mathrm{~h}$ or at $40 \mathrm{nM}$ for $0,6,12$, or $24 \mathrm{~h}$, or were exposed to the proteasome inhibitor MG132 at 0, $0.1,0.5$, or $1 \mu \mathrm{M}$ for $24 \mathrm{~h}$ or at $0.5 \mu \mathrm{M}$ for $0,6,12$, or $24 \mathrm{~h}$. 

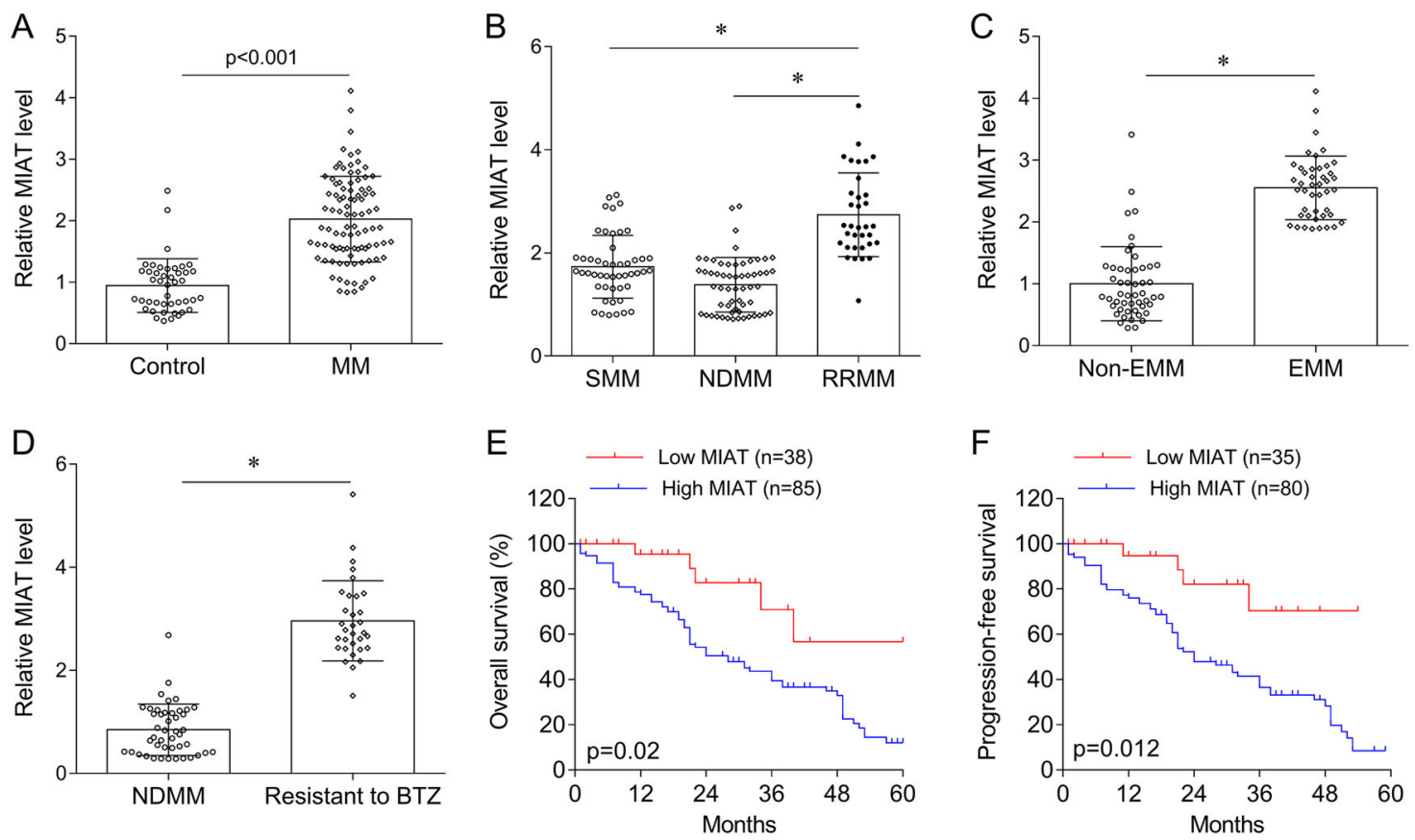

Fig. 2 Myocardial infarction associated transcript (MIAT) expression in MM. a Quantitative reverse transcriptase-polymerase chain reaction (qRTPCR $)$ analyses of MIAT expression in control $(N=56)$ and MM tissues $(N=143)$. b MIAT in smoldering MM $(S M M ; N=35)$, newly diagnosed MM $(N D M M ; N=46)$, and relapsed/refractory (RRMM; $N=34)$ patients. $c$ MIAT expression in extramedullary MM (EMM; $N=28)$ and non-EMM $(N=115)$ patients. d MIAT expression in NDMM $(N=46)$ and bortezomib (BTZ) resistant $(N=25)$ patients. e Overall survival analysis in patients with MM with low or high MIAT expression. f Progress-free survival analysis in patients with MM with low or high MIAT expression, ${ }^{*} P<0.05$; NDMM newly diagnosed MM, RRMM relapsed/refractory MM, SMM smoldering multiple myeloma, EMM extramedullary myeloma

The inhibitors BTZ, MG132, SP600125 (c-Jun Nterminal kinase, JNK), U0126 (extracellular signalregulated kinase, ERK), SB203580 (p38), Bay-11-7082 (NF-kB), and PF-04965842 (Janus kinase 1, Jak1) were purchased from Selleck (Shanghai, China). U266 cells were pretreated with these inhibitors for $2 \mathrm{~h}$ prior to further treatments and analyses.

\section{Cell infection and transfection}

An optimal MIAT sequence was selected from three shRNAs and was recombined into a lentivirus vector to produce Lv-sh-MIAT. The target sequence was ACCCT GATCATTGCAAGGATCTCGTC. Lentiviral transduction particles containing shRNA for stat1 (TRC Number: TRCN000000426) were purchased from Merck (Shanghai, China). Cells $\left(2 \times 10^{5}\right.$ cells per well $)$ were seeded into culture wells and were cultured for $24 \mathrm{~h}$. Upon reaching $80 \%$ confluence, cells were infected with lentivirus at a multiplicity of infection of 50 and were supplemented with $5-\mathrm{mg} / \mathrm{ml}$ polybrene (Sigma-Aldrich, St. Louis, MO, USA) for $48 \mathrm{~h}$.

Plasmids expressing stat1 and empty pDONR223 plasmids were purchased from Youbio Co., Ltd. (cat no. G104301, Changsha, China). A nontargeting shRNA lentivirus expression vector was used as a negative control
(Lv-sh-NC). Hsa-miR-29b inhibitors and mimics were purchased from RiboBio Co., Ltd. (Guangzhou, China) and were transfected into cells using Lipofectamine 3000 (Invitrogen) according to the manufacturer's instructions.

\section{qRT-PCR}

Total RNA was extracted using TRIzol reagent (Invitrogen). Reverse transcription was then performed using a maxima First Strand cDNA Synthesis kit (Thermo Fisher Scientific, Inc.) according to the manufacturer's protocol. Quantitative polymerase chain reactions (qPCR) were then performed using a CFX96 Touch $^{\mathrm{TM}}$ Deep Well RealTime PCR Detection System (BioRad, Hercules, CA, USA). Mature miR-29b, miR-489, and miR-150 expression levels were determined using Hairpin-it miRNAs qPCR kits (Shanghai GenePharma Co., Ltd., Shanghai, China) according to the manufacturer's protocol. Expression levels of the RNA U6 small nuclear 6 pseudogene were used as an internal control.

MIAT, Mcl-1, CDK-6, PSME4, and SP-1 expression levels were determined using UltraSYBR Mixture (CWBio, Wuhan, China) and $\beta$-actin expression was used as an internal control. Primers and their sequences are listed in Table 2. Thermocycling was performed with an initial denaturation at $95.0^{\circ} \mathrm{C}$ for $3 \mathrm{~min}$ followed by 39 
Table 3 Clinical association between MIAT levels and clinicopathological variables of patients with multiple myeloma

\begin{tabular}{|c|c|c|c|}
\hline \multirow[t]{2}{*}{ Variable } & \multicolumn{2}{|l|}{ MIAT levels } & \multirow{2}{*}{$\begin{array}{l}X^{2} \text { test } \\
P \text { value }\end{array}$} \\
\hline & $\begin{array}{l}\text { Low expression } \\
(n=38)\end{array}$ & $\begin{array}{l}\text { High expression } \\
(n=85)\end{array}$ & \\
\hline Age & & & 0.836 \\
\hline$<50$ & 13 & 27 & \\
\hline$\geq 50$ & 25 & 58 & \\
\hline Gender & & & 0.555 \\
\hline Male & 24 & 48 & \\
\hline Female & 14 & 37 & \\
\hline Cytogenetic risk (\%) & & & 0.001 \\
\hline High & 14 & 59 & \\
\hline Standard & 24 & 26 & \\
\hline Deletion 13q (\%) & & & 0.700 \\
\hline Yes & 20 & 48 & \\
\hline No & 18 & 37 & \\
\hline Deletion 17p (\%) & & & 0.845 \\
\hline Yes & 22 & 51 & \\
\hline No & 16 & 34 & \\
\hline t.(11.14) (\%) & & & 0.229 \\
\hline Yes & 17 & 28 & \\
\hline No & 21 & 57 & \\
\hline t.(14.16) (\%) & & & 0.890 \\
\hline Yes & 13 & 28 & \\
\hline No & 25 & 57 & \\
\hline DSS & & & 0.001 \\
\hline । & 15 & 12 & \\
\hline$\|$ & 16 & 34 & \\
\hline III & 7 & 39 & \\
\hline ISS & & & 0.001 \\
\hline 1 & 16 & 15 & \\
\hline$\|$ & 17 & 33 & \\
\hline III & 5 & 37 & \\
\hline $\lg H(\%)$ & & & 0.010 \\
\hline $\lg A$ & 18 & 16 & \\
\hline $\lg D$ & 0 & 4 & \\
\hline $\lg G$ & 16 & 55 & \\
\hline $\lg M$ & 1 & 6 & \\
\hline BJ & 3 & 4 & \\
\hline $\lg \mathrm{L}(\%)$ & & & 0.028 \\
\hline K & 26 & 40 & \\
\hline$\lambda$ & 12 & 45 & \\
\hline
\end{tabular}

DSS Durie-Salmon staging, ISS international staging system

cycles of $95.0^{\circ} \mathrm{C}$ for $10 \mathrm{~s}$ and $60^{\circ} \mathrm{C}$ for $30 \mathrm{~s}$. Relative gene expression was calculated using the comparative $\mathrm{Ct}$ method formula $2^{-\Delta \Delta \mathrm{Ct}}$.

\section{Western blotting}

Proteins were extracted using RIPA lysis buffer (Boster, Wuhan, China) and protein concentrations were determined using BCA protein assay kits (Thermo Scientific, Waltham, MA). Following separation on $10 \%$ sodium dodecyl sulfate-polyacrylamide gel electrophoresis (SDSPAGE) gels, proteins were transferred onto polyvinylidene (PVDF) membranes and were immunoblotted with the following primary antibodies: phospho-Stat1 (Tyr701) antibody (Rabbit monoclonal, diluted at 1:1000, cat no. 9167, Cell Signaling Technology), phospho-Stat1 (Ser727) antibody (Rabbit monoclonal, diluted at 1:1000, cat no. 8826, Cell Signaling Technology), Stat1 antibody (Rabbit monoclonal, diluted at 1:1000, cat no. 14994, Cell Signaling Technology), p38 MAPK antibody (Rabbit monoclonal, diluted at 1:1000, cat no. 8690, Cell Signaling Technology), Bcl-2 antibody (Rabbit monoclonal, diluted at 1:1000, cat no. 4223, Cell Signaling Technology), Bak1 antibody (Rabbit monoclonal, diluted at 1:1000, cat no. 12105, Cell Signaling Technology), cleaved PARP antibody (Rabbit monoclonal, diluted at 1:1000, cat no. 5625, Cell Signaling Technology), total PARP antibody (Rabbit monoclonal, diluted at 1:1000, cat no. 9532, Cell Signaling Technology), Mcl-1 antibody (Rabbit monoclonal, diluted at 1:1000, cat no. 94296, Cell Signaling Technology), CDK-6 antibody (Rabbit monoclonal, diluted at 1:1000, cat no. 13331, Cell Signaling Technology), PSME4 antibody (Rabbit polyclonal, diluted at 1:1000, cat no. ABIN5533127, Abgent Biotech. (SuZhou) Co. Ltd., Suzhou, China), SP-1 antibody (Rabbit monoclonal, diluted at 1:1000, cat no. 9389, Cell Signaling Technology), and GAPDH antibody (Rabbit polyclonal, diluted at 1:1000, cat no. 5174, Cell Signaling Technology). Membranes were then incubated with appropriate secondary antibodies and protein bands were visualized using a BioRad imaging system (Hercules, CA).

\section{Luciferase reporter assays}

The MIAT promoter was cloned into a pGL3-Basic vector using a Fast-Fusion ${ }^{\mathrm{Tm}}$ Cloning Kit (FulenGen, Guangzhou, China). After treating with BTZ or transfecting with Lv-sh-Stat1, U266 cells were co-transfected with the internal control plasmid pRL-TK (Renilla luciferase reporter plasmid, Promega) and luciferase reporter constructs. Finally, luciferase activities were determined using a Dual Luciferase Reporter Assay Kit (Promega) according to the manufacturer's instructions.

In further luciferase reporter assays, miRNAs $(n=200$, Life Technologies) or negative control RNA were cotransfected with Renilla and firefly reporters with or without MIAT. Luciferase activities were then determined using Dual Luciferase Reporter Assay Kits (Promega) according to the manufacturer's instructions.

To confirm the identities of target genes, the $3^{\prime}$ untranslated region (UTR) of miR-29b was amplified and subcloned immediately downstream of the luciferase gene sequence. Mutant MIAT (indicated by blue in Fig. 6f) was synthesized by Shanghai GenePharma Co., Ltd. Cells were then seeded and co-transfected with 100-ng aliquots of 

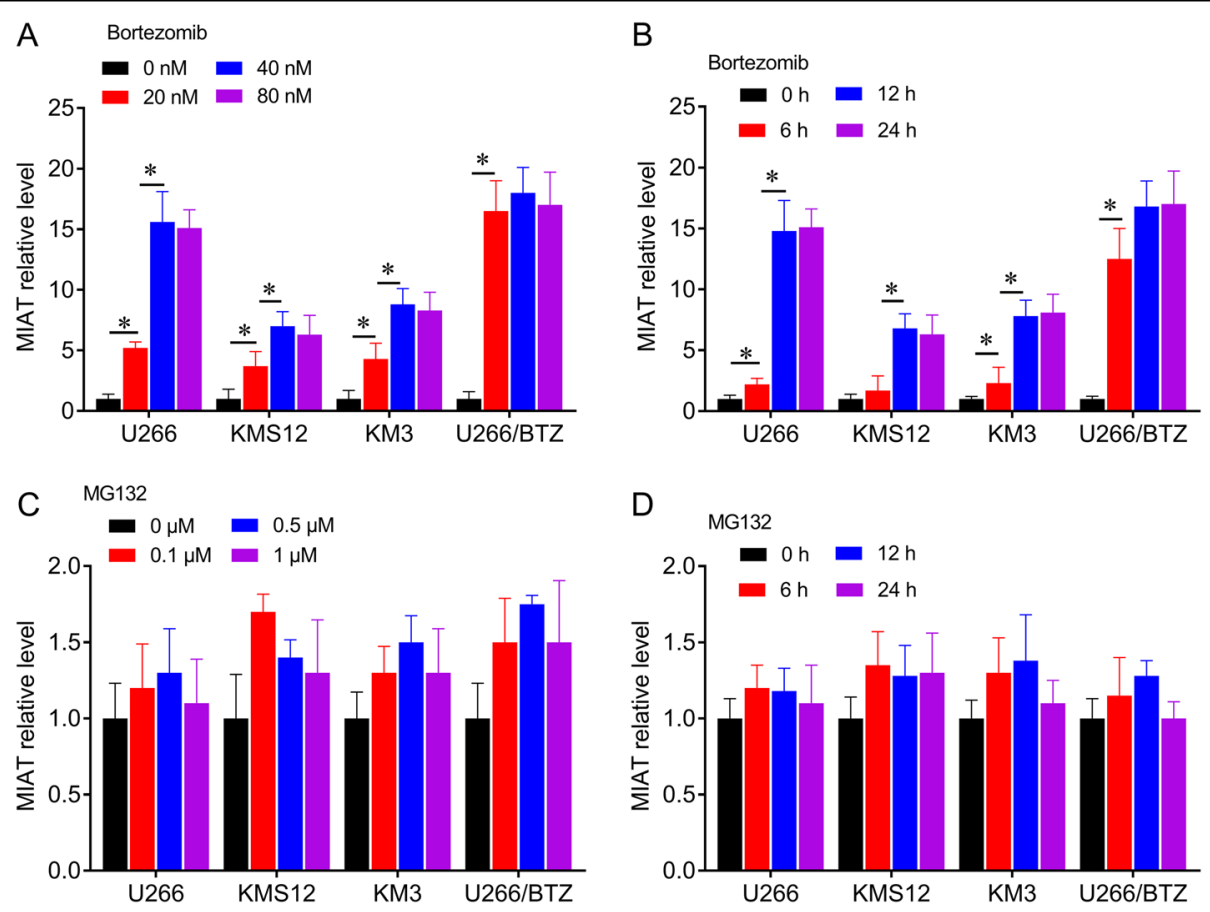

Fig. 3 Changes in MIAT expression in response to BTZ and MG132 in myeloma cell lines. a Cells were treated for 24 h with BTZ at 0, 20,40, and $80 \mathrm{nM}$. b Cells were treated with BTZ at $40 \mathrm{nM}$ for 0, 6, 12, or $24 \mathrm{~h}$. c Cells were treated for $24 \mathrm{~h}$ with MG132 at 0, 20, 40, and 80 nM. d Cells were treated with MG132 at $0.5 \mu \mathrm{M}$ for $0,6,12$, or $24 \mathrm{~h},{ }^{*} P<0.05$

constructs with or without miR-29b mimics using Lipofectamine 3000 (Thermo Fisher Scientific, Inc.). Luciferase activity was detected as described above.

\section{CCK-8 assays}

Cell viability was determined using CCK8 assays. After seeding at 5000 cells/well and culturing for $24 \mathrm{~h}$, cells were transfected with the indicated miRNAs or lentivirus vectors for $48 \mathrm{~h}$. Cells were then incubated for 24,48 , or $72 \mathrm{~h}$, and 10- $\mu \mathrm{l}$ aliquots of CCK-8 reagents (Beyotime, Hangzhou, China) were added to each well. Optical density values were then measured using a microplate reader.

\section{Apoptosis analyses}

After indicated treatments, cells were collected and washed twice in PBS and were then resuspended in 500- $\mu \mathrm{l}$ aliquots of binding buffer and incubated with $10-\mu \mathrm{l}$ aliquots of Annexin V-FITC and 10- $\mu$ l aliquots of propidium iodide (Propidium Iodide Annexin V apoptosis detection kit, Life Technologies, Grand Island, NY) for $15 \mathrm{~min}$. Finally, the cells were analyzed using flow cytometry (BD Biosciences, San Jose, CA).

\section{Chromatin immunoprecipitation (ChIP) assays}

Chromatin immunoprecipitation experiments were performed using EpiQuik Chromatin Immunoprecipitation Kits (EpiGentek, NY, USA). In these experiments, cells were lysed in RIPA lysis buffer. After centrifugation, supernatants were incubated with magnetic beads conjugated with antibodies (anti-Stat1, anti-Stat3, or anti-p65 antibody) overnight at $4{ }^{\circ} \mathrm{C}$. Normal mouse IgG (Millipore) was used as a negative control. Finally, purified DNAs from precipitates were used for $\mathrm{qPCR}$ analyses.

\section{Pulldown assays with biotinylated MIAT and biotinylated miR-29b}

Biotin labeling was performed using the Biotin RNA Labeling Mix (Roche Diagnostics, Indianapolis, IN) that was used for MIAT transcription. MIAT was then purified using RNeasy Mini Kits (Qiagen, Valencia, CA) according to the manufacturer's protocol. Subsequently, the biotinylated MIAT probe was incubated with Nanobeads M-280 Streptavidin (Invitrogen, CA, USA) to generate probe-coated beads. U266 cell lysates were then incubated with these for $12 \mathrm{~h}$ and qPCR analyses were performed after eluting RNA complexes from the beads.

Biotinylated miR-29b, miR-29b-Mut, and negative control constructs were purchased from Ribobio (Guangzhou, China) and were used to transfect U266 cells for $48 \mathrm{~h}$. After harvesting and lysing cells, lysates were incubated with Dynabeads M-280 Streptavidin (Invitrogen, CA, USA), and after elution from the beads, the RNA complexes were analyzed using qPCR. 


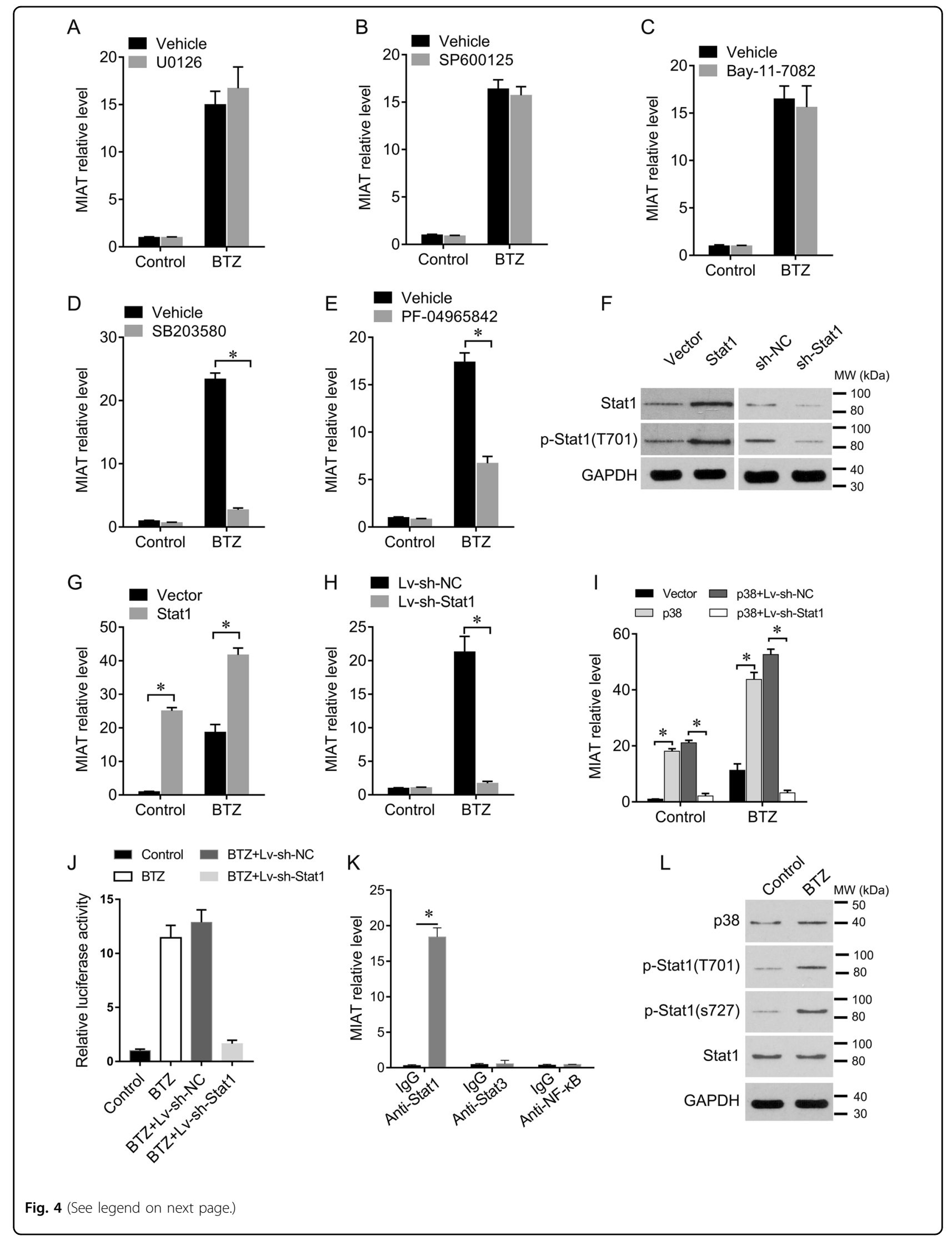


(see figure on previous page)

Fig. 4 BTZ upregulates MIAT in MM cells via p38-Stat1 signaling. a-e U266 cells were pretreated with the selective pharmacological inhibitors

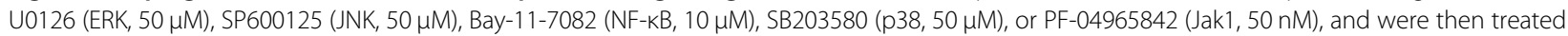
with BTZ at $40 \mathrm{nM}$ for $12 \mathrm{~h}$. MIAT expression levels were determined using qRT-PCR. $\mathbf{f}$ Western blotting analyses of Stat1 and phosphorylated Stat1 after overexpression or knockdown in U266 cells. $\mathbf{g}$ Effects of Stat1 overexpression on MIAT expression in U266 cells. $\mathbf{h}$ Effects of Stat1 knockdown on MIAT expression in U266 cells. i Effects of p38 overexpression on MIAT expression in U266 cells pretreated with sh-NC or sh-Stat1. $\mathbf{j}$ Luciferase reporter constructs containing the MIAT promoter were co-transfected into U266 cells with the internal control plasmid pRL-TK, and with sh-NC or sh-Stat1, and were then subjected to BTZ challenge $(40 \mathrm{nM}, 12 \mathrm{~h})$. Relative luciferase activities are expressed as percentages of those in the control group. $\mathbf{k}$ Cell lysates from U266 cells were used for RIP with antibodies against stat1, stat3, or NF-kB. MIAT expression levels were detected using qRT-PCR. IgG was used as a negative control. I BTZ induced Stat1 phosphorylation; data are presented as means \pm standard errors of the mean from three independent experiments; ${ }^{*} P<0.05$; two-tailed pairwise comparisons were made with Student's $t$-test

\section{Tumor xenograft mice}

All animal experiments were approved by the Ethics Committee for Animal Research of the Third Xiangya Hospital of Central South University. Mice were randomly divided into two groups and were injected with negative control transfected cells or sh-MIAT transfected cells by researchers who were blinded to the treatments. Transfected U266 cells $\left(1 \times 10^{6}\right)$ were then subcutaneously injected into nude mice $(N=5)$ and tumor sizes were recorded periodically. Tumor volumes were calculated as $0.5 \times L \times W^{2}$, where $L$ and $W$ are long and short diameters of the tumor mass, respectively.

\section{Statistical analysis}

Statistical analyses were performed using GraphPad Prism software (GraphPad Software Inc., La Jolla, CA). Significant differences were identified using Student's $t$-test, one-way ANOVA with Bonferroni tests, Kaplan-Meier with log-rank tests, and Pearson's correlation analyses, and were considered significant when $P<0.05$.

\section{Results}

\section{Differentially expressed IncRNAs in MM tissues}

To identify lncRNAs that are involved in MM development, we performed microarray analyses of bone marrow (BM) tissues from patients with $\mathrm{MM}$ and from healthy donors. Differentially expressed lncRNAs, including 1661 upregulated genes (red plots) and 1489 downregulated genes (green plots), are presented in volcano plots (Fig. 1a-c). IncRNA-MIAT was highly upregulated in BM tissues. Additionally, we analyzed the pathways involved in these lncRNAs. These differentially expressed lncRNAs were enriched in DNA replication, nucleotide excision repair, cell cycle, and carbon metabolism (Fig. 1d).

\section{MIAT expression is higher in MM plasma cells than in control cells}

MIAT expression was significantly higher in $M M$ plasma cells than in those from healthy controls $(P<$ 0.001; Fig. 2a), and was also higher in patients with
RRMM than in NDMM $(P<0.05)$ and SMM $(P<0.05)$ patients (Fig. 2b). MIAT expression in intramedullary plasma cells was higher in MM with EMM than in those without EMM $(P<0.05$; Fig. 2c). MIAT expression was also significantly increased in patients with $\mathrm{MM}$ with BTZ resistance $(P<0.05$, Fig. $2 d)$. After dividing patients with MM into high and low MIAT expression groups using the median MIAT expression as a cut off, MIAT expression levels were significantly associated with cytogenetic risk $(P=0.001)$, DSS $(P=0.001)$, ISS $(P=0.001)$, IgH $(P=$ $0.023)$, and $\operatorname{IgL}(P=0.043)$, but were not associated with gender, age, or gene deletion and translocation (Table 3). Furthermore, in clinical assessments of the impact of MIAT expression in high and low MIAT expression groups from 123 patients with MM with overall survival (OS) data and 115 patients with MM with unavailable data, high MIAT expression was significantly associated with worse prognosis (Fig. 2e, f).

\section{MIAT is a BTZ-inducible IncRNA in MM cells}

Because MIAT expression was greater in BTZ-resistant patients with MM than in NDMM patients, we confirmed responses of MIAT to BTZ using qRT-PCR analyses of MM cells. MIAT expression was also induced by BTZ in U266, KMS12, KM3, and U266/BTZ cells, and peaked at $12 \mathrm{~h}$ in the presence of $40-\mathrm{nM}$ BTZ (Fig. 3a, b). In contrast, MG132 treatments did not lead to significant changes in MIAT expression in any of the cell lines (Fig. $3 c, d)$.

\section{Upstream mediators of MIAT expression in MM cells}

To distinguish signaling pathways through which BTZ inhibits MM cell growth, we performed experiments with various pathway inhibitors. Pretreatment with the ERK inhibitor SB203580 completely abrogated BTZ-mediated induction of MIAT, which was also partly reversed by the Jak1 inhibitor PF-04965842 (Fig. 4a-e).

Stat1 overexpression (Fig. 4f) also significantly enhanced BTZ-induced MIAT expression, whereas silencing of Stat1 suppressed BTZ-induced MIAT expression (Fig. 4g, h). Congruently, p38 overexpression 

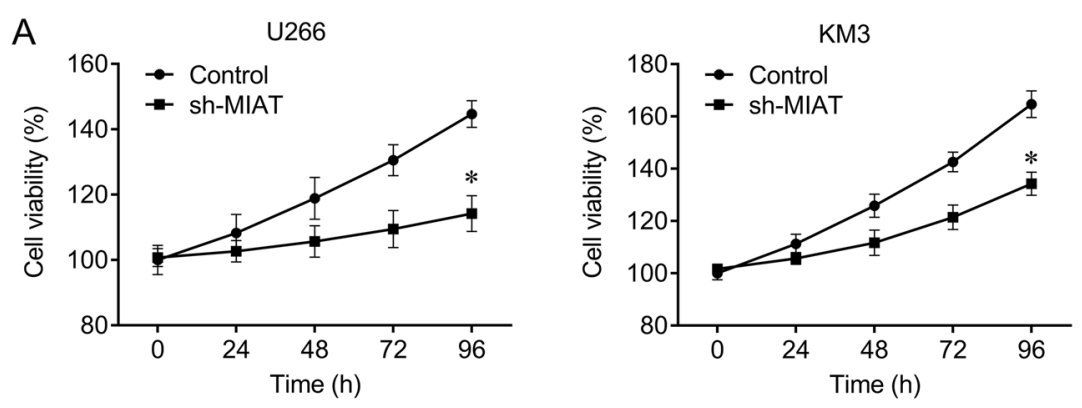

B
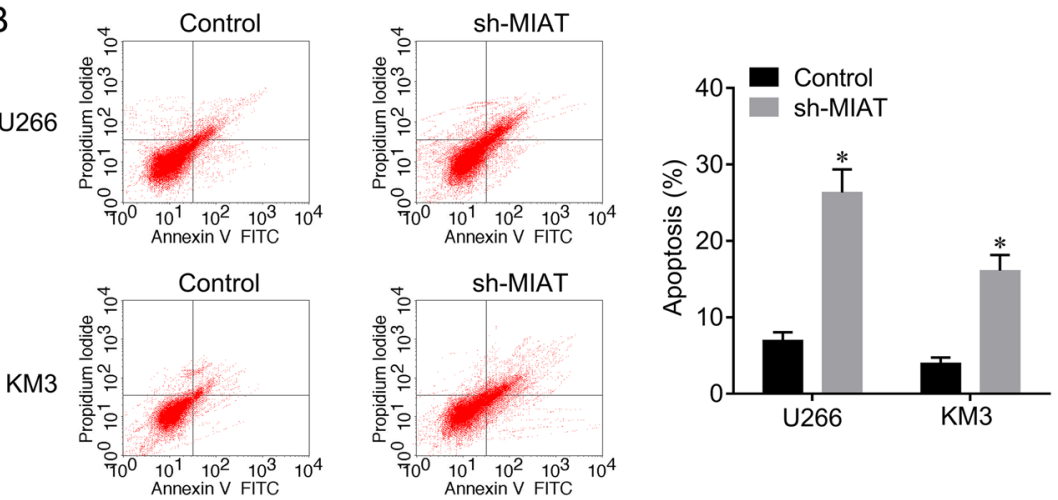

C
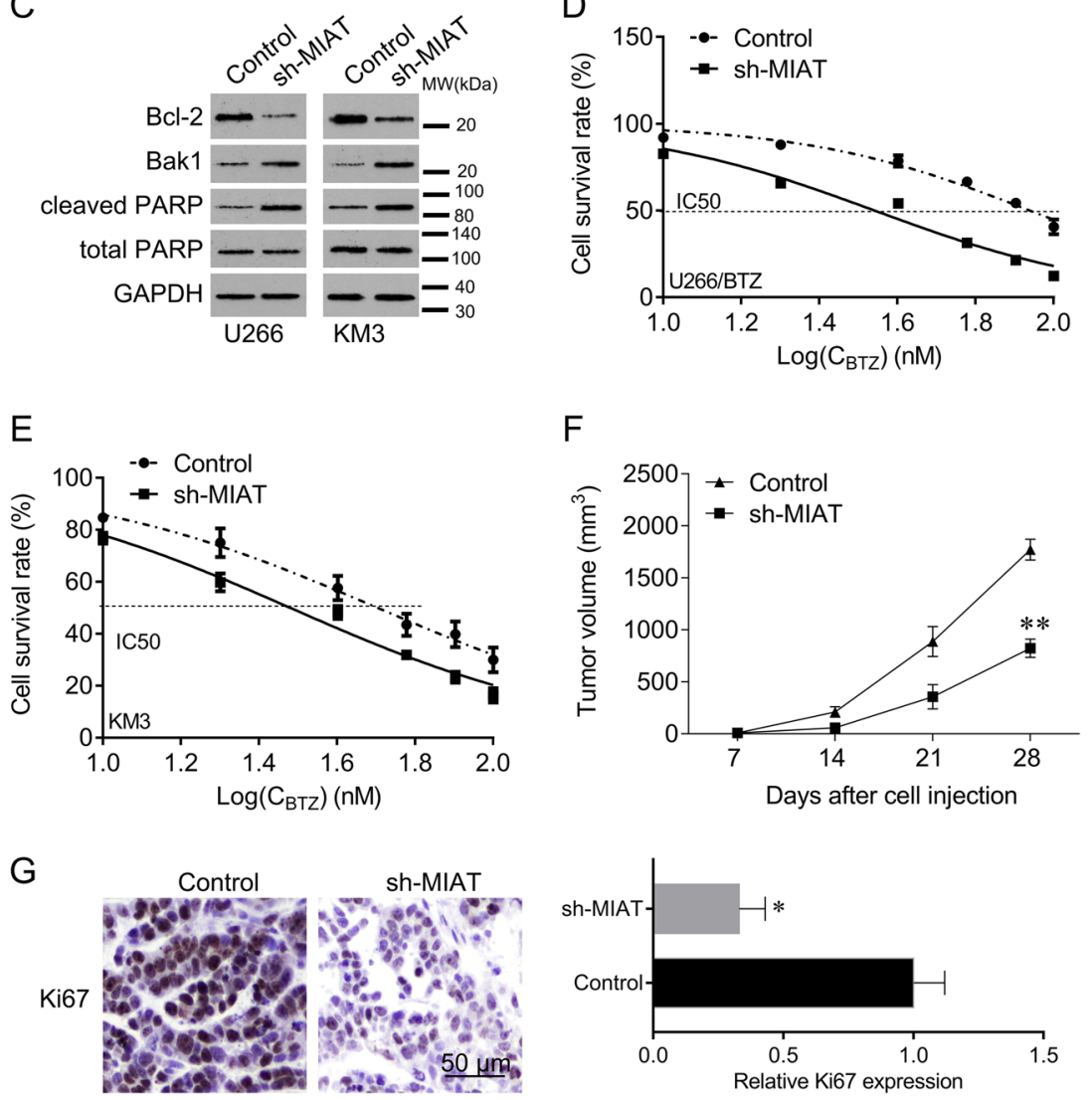

Fig. 5 (See legend on next page.) 
(see figure on previous page)

Fig. $\mathbf{5}$ Knockdown of MIAT sensitizes MM cells to BTZ. a CCK-8 assays were used to determine cell viability after sh-MIAT lentivirus infection. $\mathbf{b}$ Flow cytometry was used to determine apoptosis after knockdown of MIAT. c Western blot analyses of apoptosis markers Bcl-2, Bak1, and cleaved PARP after knockdown of MIAT. $\mathbf{d}$ Changes of in half inhibitory concentrations $\left(I C_{50}\right)$ of BTZ in U266 cells after knockdown of MIAT were determined using CCK-8 assays. e Changes in IC 50 of BTZ in KM3 cells after knockdown of MIAT. $\mathbf{f} U 266$ cells were infected with MIAT shRNA lentivirus and were then injected into nude mice. Cells transfected with empty lentivirus were used as a negative control. Mice were euthanized and tumors were collected on day 28 after injection. Tumor volumes were measured every week. g Ki67 expression in tumor sections was evaluated using immunohistochemistry (IHC); Bar, $50 \mu \mathrm{m} ;{ }^{*} P<0.05,{ }^{*} P<0.01$

increased MIAT induction by BTZ, and this effect was reversed by silencing Stat1 (Fig. 2i). BTZ treatments also significantly increased MIAT promoter activity, and Stat1 knockdown abrogated this effect (Fig. 4j). In addition, ChIP results showed that MIAT interacts with Stat1 but not with Stat3 and NF-KB (Fig. 3h). In accordance, BTZ treatments increased Stat1 phosphorylation at serine 727 and tyrosine 701 (Fig. 4l).

\section{MIAT knockdown inhibits MM cell growth}

MIAT knockdown significantly inhibited cell proliferation and promoted apoptosis compared with that in the negative control (Fig. 5a, b). MIAT knockdown also reduced $\mathrm{Bcl}-2$ expression and increased $\mathrm{Bak} 1$ and cleaved PARP expression (Fig. 5c). In addition, the half inhibitory concentrations $\left(\mathrm{IC}_{50}\right.$ ) of BTZ in U266/BTZ and KM3 cells were significantly reduced by sh-MIAT lentivirus infection, compared with those in the negative control (Fig. 5d, e). Furthermore, MIAT knockdown significantly inhibited tumor growth in vivo and decreased the expression of the proliferation marker Ki67 (Fig. 5f, g). These data collectively indicate that knockdown of MIAT inhibits cell growth and mitigates BTZ resistance in MM cells.

\section{MIAT interacts with miR-29b and negatively regulates its expression}

The miRNAs miR-29b, miR-489, and miR-150 had low luciferase activities and were screened as targets of MIAT (Fig. 6a). In correlation analyses of their expression levels and MIAT levels, only miR-29b was negatively correlated with MIAT (Fig. 6b-d). In addition, knockdown of MIAT significantly increased miR-29b expression but not that of miR-489 and miR-150 (Fig. 6e), further confirming the target relationship. Upregulation of miR-29b also decreased wild type (WT) MIAT-luciferase activity, but had no effect on mutant (MUT) MIAT luciferase activity (Fig. 6g). Accordingly, WT MIAT pulled miR-29b down, whereas MUT MIAT did not (Fig. 6h, i). Congruently, miR-29b expression was significantly decreased by WT MIAT but not by MUT MIAT in U266 cells (Fig. 6j). In further analyses of miR-29b target genes, we showed that knockdown of MIAT significantly reduces the expression of Mcl-1, CDK-6, PSME4, and SP-1 compared with controls, and an inhibitor of miR-29b reversed the reductions of Mcl-1, CDK-6, PSME4, and SP-1 that followed MIAT downregulation (Fig. 6k, l). These results suggest that MIAT functions as a miR-29b sponge and negatively regulates its expression in $\mathrm{MM}$ cells, leading to control over the expression of miR-29b target genes.

\section{MIAT silencing sensitizes MM cells to BTZ through miR-29b}

Downregulated miR-29b repressed MIAT shRNAmediated apoptosis (Fig. 7a). In addition, MIAT knockdown reduced the $\mathrm{IC}_{50}$ of $\mathrm{BTZ}$ in U266 cells, whereas the miR-29b inhibitor reversed this decrease (Fig. 7b). The results indicate that MIAT enhances BTZ resistance in MM cells by targeting miR-29b.

\section{Discussion}

Our previous studies show that MIAT expression is upregulated in lung cancer tissues compared with adjacent tissues. In particular, increased MIAT expression was associated with advanced stages and shorter OS times of lung cancer patients ${ }^{13}$. The present data show that higher MIAT expression is predictive of reduced survival of patients with MM. MIAT expression was also significantly higher in BTZ-resistant patients with MM than in newly diagnosed patients with MM, and MIAT was identified as a BTZ-inducible lncRNA. We also show that BTZ upregulates MIAT expression by increasing the phosphorylation of stat1. Conversely, silencing of MIAT inhibited MM cell growth and sensitized MM cells to BTZ by negatively regulating miR-29b.

MIAT (located at 22q12.1) was originally identified as a myocardial infarction susceptibility locus ${ }^{14}$, and its expression was reportedly upregulated in association with ischemic stroke, myocardial infarction, non-small-cell lung cancer, and chronic lymphocytic leukemia ${ }^{15,16}$. Hence, MIAT may provide a therapeutic target for several cancers, including prostate and non-small-cell lung cancers $^{17,18}$. In other studies, MIAT was identified as an oncogenic lncRNA that promoted colorectal and gastric cancers by targeting miR-132 and miR-141 ${ }^{19,20}$. Our data show for the first time that MIAT is highly expressed in patients with MM and that BTZ treatment induces MIAT expression.

In previous investigations, the antitumor effects of BTZ were mediated by several pathways, including those 


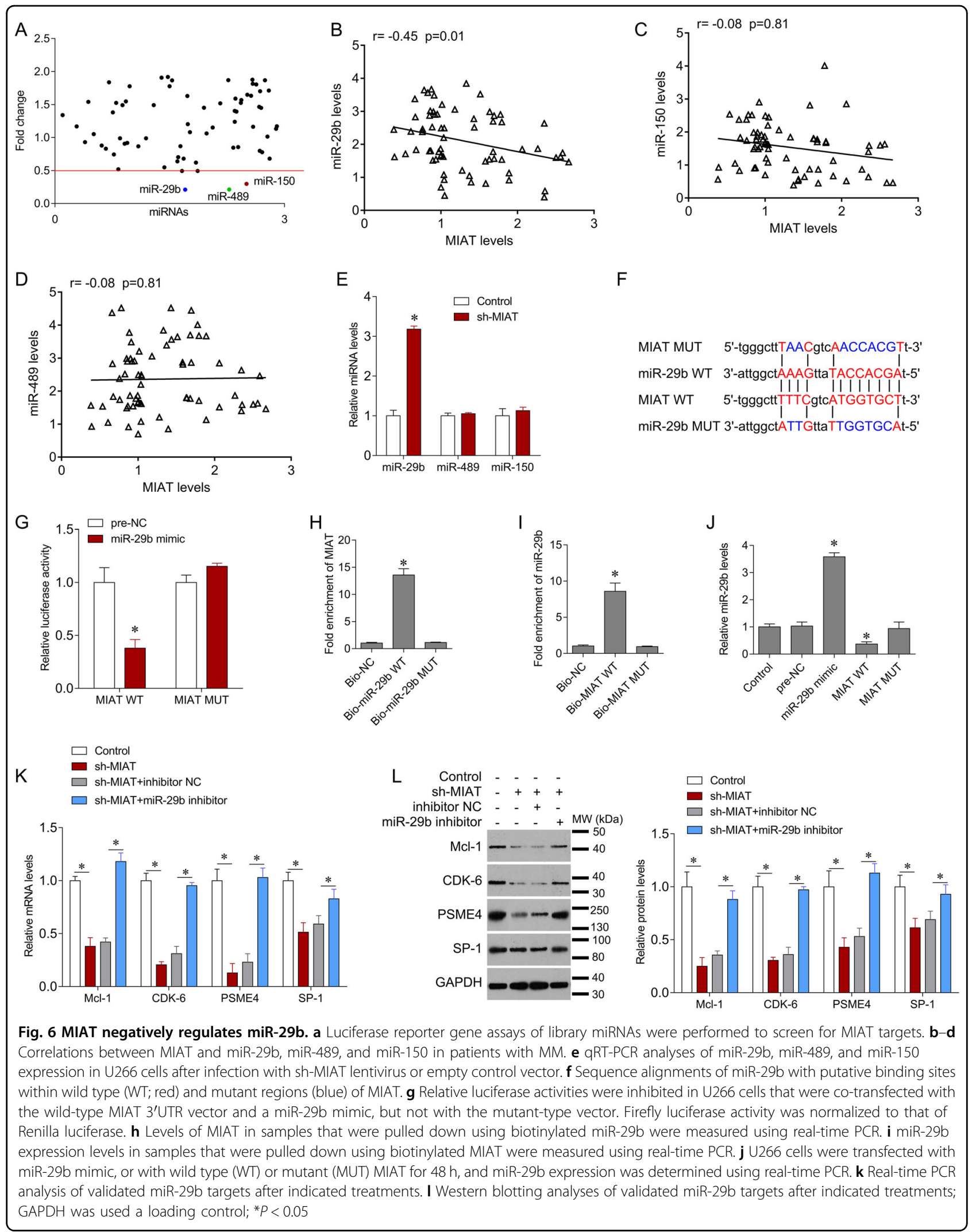



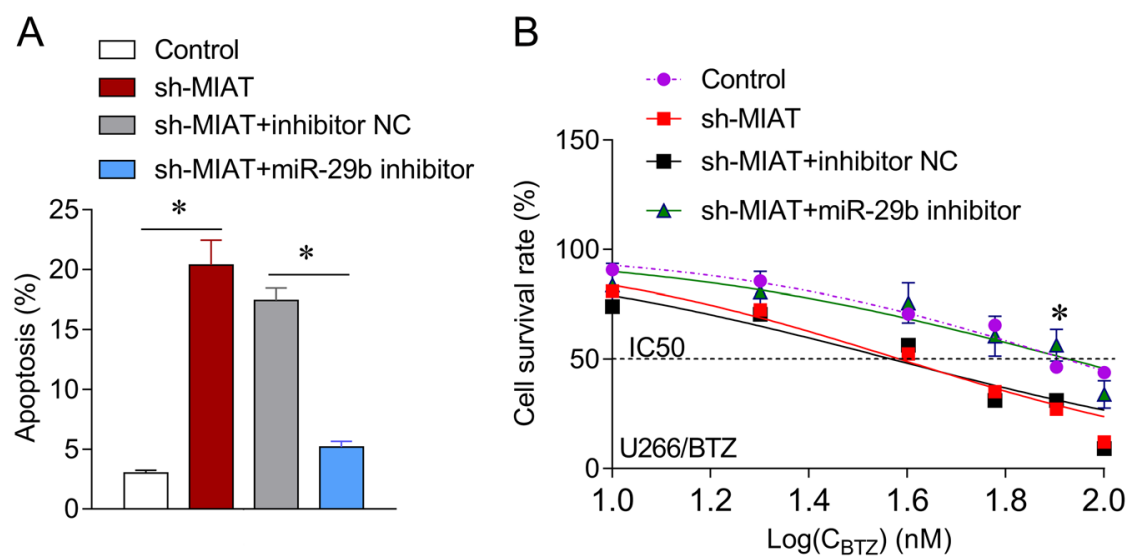

Fig. 7 miR-29b inhibitor reverses the inhibitory effects of MIAT downregulation in U266 cells. a Apoptosis was evaluated using flow cytometry after treating U266 cells with miR-29b inhibitor and MIAT shRNA. b Changes in IC 50 values for BTZ in U266 cells were determined using CCK-8 assays after treatments with miR-29b inhibitor and MIAT shRNA; ${ }^{P} P<0.05$

involving NF-kB, ERK, and $\mathrm{JNK}^{21-23}$. Moreover, BTZ inhibited both canonical and noncanonical activation of $\mathrm{NF}-\mathrm{kB}$ in MM cells. Yet, atypical NF- $\mathrm{kB}$ activation can promote BTZ resistance in MM cells ${ }^{24}$. Nonetheless, we found that co-treatments with BTZ and inhibitors of NF$\kappa B$, ERK, or JNK did not affect MIAT expression. But cotreatment with BTZ and a p38 inhibitor significantly suppressed MIAT expression, and the Jak1 inhibitor partially reversed BTZ-induced MIAT expression. Hence, BTZ resistance may result in constitutive activation of ERK, NF-кB, and stat1 pathways ${ }^{25,26}$. Similarly, p38 may promote drug resistance in patients with $M M$ by regulating ERK, NF-KB, and stat1 pathways ${ }^{27,28}$. Constitutive nuclear localization and transcription of Stat1 activates downstream target genes. In a study by Shen et al., stat 1 mediated LINC00174 expression in colorectal carcino$\operatorname{mas}^{29}$. Stat1 also interacts with lncRNA PLAC2 to inhibit cell proliferation and induce cell cycle arrest in gliomacells $^{30}$. Herein, we concordantly demonstrated that stat 1 interacts with the MIAT promoter and positively regulates MIAT expression.

Silencing of MIAT inhibited MM cell growth and sensitized MM cells to BTZ. According to the competitive endogenous RNA (ceRNA) hypothesis, mRNAs and lncRNAs communicate with each other by competing for binding sites on miRNAs. Congruently, Li et al. found that MIAT binds to miR-29-3p and upregulates the expression of HDAC4 ${ }^{31}$. Our recent studies also showed that MIAT levels are negatively correlated with miR-29b in patients with MM, and MIAT inhibited miR-29b expression through a direct interaction, leading to increased expression of the miR-29b target genes Mcl-1, CDK-6, PSME4, and SP-1. The human miR-29b family includes miR-29a, miR-29b, and miR-29c, which are highly homologues in humans, mice, and rats $^{32}$. The anti-MM activity of miR-29b has been studied extensively ${ }^{33}$, and its expression is reportedly decreased in primary malignant plasma cells and MM cell lines ${ }^{34}$. Moreover, enforced expression of miR-29b triggered in vitro anti-MM activity by targeting MCL1 and CDK6 $6^{35,36}$. PSME4 encodes the proteasome activator PA200, and was also identified as a novel target of miR-29b in MM cells, and induction of miR-29b reversed PA200-reduced proteasome inhibition following treatments with $\mathrm{BTZ}^{37}$. In other studies, high SP1 activity was related to sustained survival and proliferation of $\mathrm{MM}^{38}$, and pharmacological or genetic inhibition of SP1 activated miR-29b transcription ${ }^{39}$. Notably, BTZ upregulates miR-29b ${ }^{34}$. In the present study, overexpressed miR-29b strongly increased growth inhibition and apoptosis in BTZ-treated MM cells, whereas miR-29b inhibition attenuated the anti-MM activity of $\mathrm{BTZ}^{34}$. In this study, we demonstrate a novel mechanism by which BTZ increases miR-29b expression. In particular, we show that BTZ induces MIAT through the p38-stat1 pathway. We further identified MIAT as a ceRNA for miR-29b and show that miR-29b inhibition attenuates sh-MIAT antiMM activity. However, further investigations are required to clarify the mechanisms by which MIAT regulates miR$29 \mathrm{~b}$ expression in MM, and these may be epigenetic, as shown in a recent study of MIAT and miR-34a expres$\operatorname{sion}^{13}$. A similar report shows that miR-29b levels are epigenetically regulated in hematologic malignancies, including $\mathrm{MM}^{40}$, further suggesting that epigenetic mechanisms contribute to the regulatory relationship between MIAT and miR-29b.

In summary, the data presented herein demonstrates that MIAT could be exploited as a tool for mitigating BTZ resistance in patients with MM. 


\section{Acknowledgements}

This work was supported by the National Natural Science Foundation of China (No. 81670203) and the Natural Science Foundation of Hunan Provincial China (Nos. 2018JJ3776 and 2017JJ3463).

\section{Authors' contributions}

J.L. and Y.L. conceived the study and participated in the study design, performance, coordination, and manuscript writing. Y.F., X.L., F.Z., and S.J. performed the research. All authors have read and approved the final manuscript In Fig. 1, J.L. generated the data and assembled the figure. In Fig. 2, Y.F. generated the data and labeled the image, Y.L. assembled the figure. In Fig. 3, Y.F. generated the data and labeled the image, Y.L. assembled the figure. In Fig. 4, X.L. and F.Z. generated the data and labeled the image, Y.L. assembled the figure. In Fig. 5, X.L. and F.Z. generated the western blot, in vivo experiments and $\mathrm{QPCR}$ data, and labeled the image, Y.L. and Y.F. assembled the figure. In Fig. 6, X.L., S.J., and F.Z. generated the western blot, qPCR and data analysis, and labeled the image, J.L., Y. L., and Y.F. assembled the figure. In Fig. 7, X.L. generated the flow cytometry data and labeled the image, Y.L. assembled the figure.

\section{Conflict of interest}

The authors declare that they have no conflict of interest.

\section{Publisher's note}

Springer Nature remains neutral with regard to jurisdictional claims in published maps and institutional affiliations.

Received: 31 January 2019 Revised: 23 March 2019 Accepted: 26 March 2019

Published online: 09 April 2019

\section{References}

1. Lipchick, B. C., Fink, E. E. \& Nikiforov, M. A. Oxidative stress and proteasome inhibitors in multiple myeloma. Pharmacol. Res. 105, 210-215 (2016).

2. Gandolfi, S. et al. The proteasome and proteasome inhibitors in multiple myeloma. Cancer Metastasis Rev. 36, 561-584 (2017).

3. Mohan, M., Matin, A. \& Davies, F. E. Update on the optimal use of bortezomib in the treatment of multiple myeloma. Cancer Manag. Res. 9, 51-63 (2017).

4. Fall, D. J. et al. Utilization of translational bioinformatics to identify nove biomarkers of bortezomib resistance in multiple myeloma. J. Cancer $\mathbf{5}$, 720-727 (2014)

5. Yang, X. et al. IncRNA PDIA3P interacts with c-Myc to regulate cell proliferation via induction of pentose phosphate pathway in multiple myeloma. Biochem. Biophys. Res. Commun. 498, 207-213 (2018).

6. Ronchetti, D. et al. A compendium of long non-coding RNAs transcriptional fingerprint in multiple myeloma. Sci. Rep. 8, 6557 (2018).

7. Handa, H. et al. Long non-coding RNA MALAT1 is an inducible stress response gene associated with extramedullary spread and poor prognosis of multiple myeloma. Br. J. Haematol. 179, 449-460 (2017).

8. Amodio, N. et al. Drugging the IncRNA MALAT1 via LNA gapmeR ASO inhibits gene expression of proteasome subunits and triggers anti-multiple myeloma activity. Leukemia 32, 1948-1957 (2018).

9. Chen, L., Hu, N., Wang, C., Zhao, H. \& Gu, Y. Long non-coding RNA CCAT1 promotes multiple myeloma progression by acting as a molecular sponge of miR-181a-5p to modulate HOXA1 expression. Cell Cycle 17, 319-329 (2018).

10. Wu, Y. \& Wang, H. IncRNA NEAT1 promotes dexamethasone resistance in multiple myeloma by targeting miR-193a/MCL1 pathway. J. Biochem. Mol. Toxicol. 32, e22008 (2018).

11. Sun, $Y$. et al. Knockdown of long non-coding RNA H19 inhibits multiple myeloma cell growth via NF-kappaB pathway. Sci. Rep. 7, 18079 (2017).

12. Lu, D., Yang, C., Zhang, Z., Cong, Y. \& Xiao, M. Knockdown of Linc00515 inhibits multiple myeloma autophagy and chemoresistance by upregulating miR-1405p and downregulating ATG14. Cell. Physiol. Biochem. 48, 2517-2527 (2018).

13. Fu, Y. et al. Silencing of long non-coding RNA MIAT sensitizes lung cancer cells to gefitinib by epigenetically regulating miR-34a. Front. Pharmacol. 9, 82 (2018).

14. Ishii, N. et al. Identification of a novel non-coding RNA, MIAT, that confers risk of myocardial infarction. J. Hum. Genet. 51, 1087-1099 (2006).
15. Zhou, X. et al. IncRNA MIAT functions as a competing endogenous RNA to upregulate DAPK2 by sponging miR-22-3p in diabetic cardiomyopathy. Cell Death Dis. 8, e2929 (2017).

16. Yan, B. et al. IncRNA-MIAT regulates microvascular dysfunction by functioning as a competing endogenous RNA. Circ. Res. 116, 1143-1156 (2015).

17. Crea, F. et al. The role of epigenetics and long noncoding RNA MIAT in neuroendocrine prostate cancer. Epigenomics 8, 721-731 (2016).

18. Lai, I. L. et al. Long noncoding RNA MIAT promotes non-small cell lung cancer proliferation and metastasis through MMP9 activation. Oncotarget 8, 98148-98162 (2017).

19. Liu, Z. et al. Long non-coding RNA MIAT promotes growth and metastasis of colorectal cancer cells through regulation of miR-132/Derlin-1 pathway. Cancer Cell Int. 18, 59 (2018).

20. Sha, M. et al. Long non-coding RNA MIAT promotes gastric cancer growth and metastasis through regulation of miR-141/DDX5 pathway. J. Exp. Clin. Cancer Res. 37, 58 (2018).

21. Fuchs, O. Targeting of NF-kappaB signaling pathway, other signaling pathways and epigenetics in therapy of multiple myeloma. Cardiovasc. Hematol. Disord. Drug Targets 13, 16-34 (2013).

22. Bai, Q. X. \& Zhang, X. Y. Curcumin enhances cytotoxic effects of bortezomib in human multiple myeloma $\mathrm{H} 929$ cells: potential roles of NF-kappaB/JNK. Int. J. Mol. Sci. 13, 4831-4838 (2012).

23. Morelli, M. B. et al. The effects of cannabidiol and its synergism with bortezomib in multiple myeloma cell lines. A role for transient receptor potential vanilloid type-2. Int. J. Cancer 134, 2534-2546 (2014).

24. Huynh, M. et al. Hyaluronan and proteoglycan link protein 1 (HAPLN1) activates bortezomib-resistant NF-kappaB activity and increases drug resistance in multiple myeloma. J. Biol. Chem. 293, 2452-2465 (2018).

25. Sagawa, M. et al. TM-233, a novel analog of 1'-acetoxychavicol acetate, induces cell death in myeloma cells by inhibiting both JAK/STAT and proteasome activities. Cancer Sci. 106, 438-446 (2015).

26. Tagoug, I., Plesa, A. \& Dumontet, C. Bortezomib influences the expression of malignant plasma cells membrane antigens. Eur. J. Pharmacol. 706, 11-16 (2013).

27. de Oliveira, M. B. et al. Anti-myeloma effects of ruxolitinib combined with bortezomib and lenalidomide: a rationale for JAK/STAT pathway inhibition in myeloma patients. Cancer Lett. 403, 206-215 (2017).

28. Peng, $H$. et al. Characterization of p38 MAPK isoforms for drug resistance study using systems biology approach. Bioinformatics 30, 1899-1907 (2014).

29. Shen, Y., Gao, X., Tan, W. \& Xu, T. STAT1-mediated upregulation of InCRNA LINC00174 functions a ceRNA for miR-1910-3p to facilitate colorectal carcinoma progression through regulation of TAZ. Gene $\mathbf{6 6 6}$, 64-71 (2018).

30. Hu, Y. W. et al. IncRNA PLAC2 down-regulates RPL36 expression and blocks cell cycle progression in glioma through a mechanism involving STAT1. J. Cell. Mol. Med. 22, 497-510 (2018).

31. Li, Y. et al. IncRNA-MIAT regulates cell biological behaviors in gastric cancer through a mechanism involving the miR-29a-3p/HDAC4 axis. Oncol. Rep. 38 3465-3472 (2017)

32. Lagos-Quintana, M., Rauhut, R., Lendeckel, W. \& Tuschl, T. Identification of novel genes coding for small expressed RNAs. Science 294, 853-858 (2001).

33. Kollinerova, S., Vassanelli, S. \& Modriansky, M. The role of miR-29 family members in malignant hematopoiesis. Biomed. Pap. Med. Fac. Univ. Palacky Olomouc Czech Repub. 158, 489-501 (2014).

34. Amodio, N. et al. miR-29b sensitizes multiple myeloma cells to bortezomibinduced apoptosis through the activation of a feedback loop with the transcription factor Sp1. Cell Death Dis. 3, e436 (2012).

35. Zhao, J. J. et al. microRNA expression profile and identification of miR-29 as a prognostic marker and pathogenetic factor by targeting CDK6 in mantle cell lymphoma. Blood 115, 2630-2639 (2010).

36. Mott, J. L., Kobayashi, S., Bronk, S. F. \& Gores, G. J. miR-29 regulates Mcl-1 protein expression and apoptosis. Oncogene 26, 6133-6140 (2007).

37. Jagannathan, S. et al. miR-29b replacement inhibits proteasomes and disrupts aggresome+autophagosome formation to enhance the antimyeloma benefit of bortezomib. Leukemia 29, 727-738 (2015).

38. Fulciniti, M. et al. Significant biological role of sp1 transactivation in multiple myeloma. Clin. Cancer Res. 17, 6500-6509 (2011).

39. Liu, S. et al. Sp1/NFkappaB/HDAC/miR-29b regulatory network in KIT-driven myeloid leukemia. Cancer Cell 17, 333-347 (2010).

40. Amodio, N. et al. miR-29s: a family of epi-miRNAs with therapeutic implications in hematologic malignancies. Oncotarget 6, 12837-12861 (2015). 